\title{
Why Clays Swell
}

\author{
Emiel J. M. Hensen* ${ }^{* \dagger}$ and Berend Smit \\ University of Amsterdam, Nieuwe Achtergracht 166, 1018 WV Amsterdam, The Netherlands
}

Received: July 10, 2002; In Final Form: September 13, 2002

\begin{abstract}
The particularly difficult subject of predicting the swelling behavior of clay minerals is addressed by a combination of molecular dynamics and Monte Carlo sampling techniques. The introduced algorithm essentially mimics the experimental determination of the water adsorption isotherm and quantitatively predicts clay swelling for a montmorillonite-type clay including such details as the occurrence of hydrated states and hysteresis. Furthermore, important insights into the underlying mechanism of clay swelling from the one-layer to the two-layer hydrate are derived. It turns out that, for this case, clay swelling proceeds via the migration of counterions that are initially bound to the mineral surface to the central interlayer plane where they become fully hydrated. The extent of clay swelling strongly depends on the charge locus. This information appears to be transferable to other clay types.
\end{abstract}

\section{Introduction}

Clays are ubiquitous minerals that figure as liner materials in the containment of waste, ${ }^{1,2}$ drug and agrochemical delivery agents, and catalytic materials. ${ }^{3}$ They also play a vital role in biogeochemical processes by retaining and releasing metal nutrients in soil. ${ }^{4}$ Whereas clays are used to the benefit as drilling mud lubricants during oil extraction, the replacement of oilbased by water-based muds induces unwanted swelling in clayrich shales and coherently mudrock damage or even well collapse. Clays consist of negatively charged aluminosilicate layers kept together by cations. The most characteristic property is their ability to adsorb water between the layers, resulting in strong repulsive forces and clay expansion. Because the properties of a liquid near a surface are totally different from those of the bulk liquid, macroscopic theories such as DLVO theory have failed to describe typical stepwise microscopic swelling. In this regime, swelling strongly depends on the molecular packing of intercalated water ${ }^{5}$ and statistical-mechanical simulations have unveiled many details of the confined fluid's structure, ${ }^{6-15}$ but not of the swelling mechanism due to their limitations to predict clay water content. Here we employ modern Monte Carlo methods, which enable us to compute water adsorption isotherms for sodium montmorillonite clay. Moreover, the simulations give us detailed insights into the swelling mechanism of this prototypical swelling clay which are transferable to other types of clay.

\section{Clay Water Adsorption Isotherms}

Typical for microscopic or crystalline swelling is that this process occurs in discrete steps, and one observes the occurrence of one, two, or three water layers in the clay interlayer. Figure 1 shows the atomistic model of the one-layer hydrate of sodium montmorillonite including details of the used algorithm. For a water vapor pressure of $p / p_{0}=0.3$, our simulation code predicts the occurrence of this one-layer hydrate with a spacing of 12.3 $\AA$, which is in excellent agreement with experimental values. ${ }^{16-21}$

* Corresponding author. E-mail: E.J.M.Hensen@TUE.nl

$\dagger$ Present address: Schuit Institute of Catalysis, Eindhoven University of Technology, P.O. Box 513, 5600 MB Eindhoven, The Netherlands.

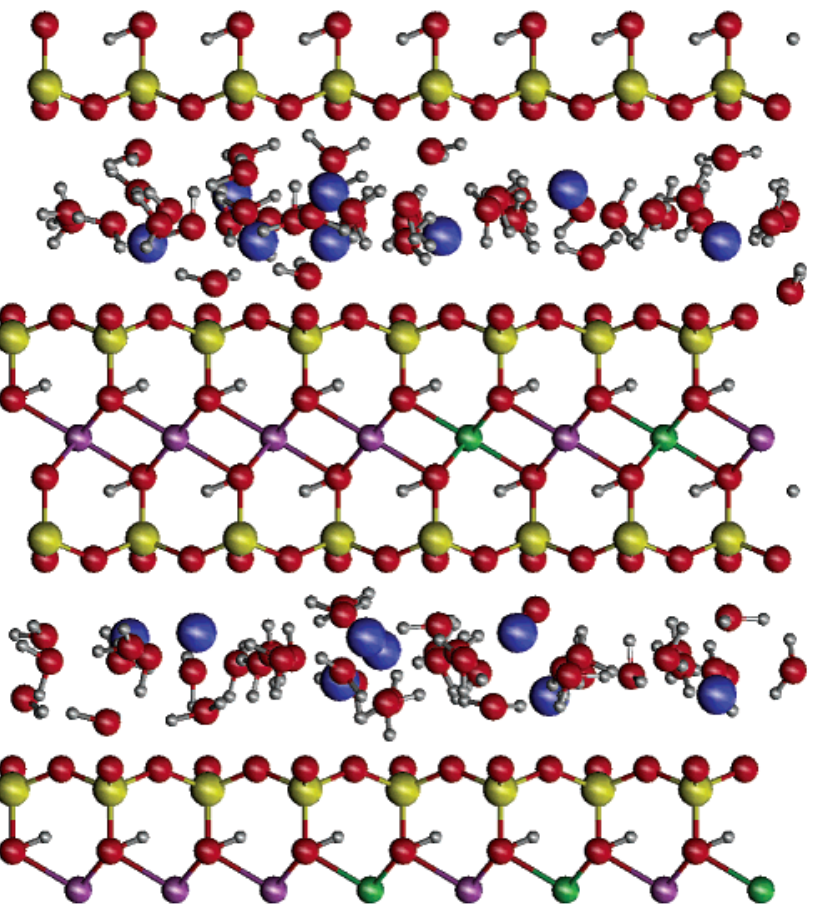

Figure 1. Atomistic model of the one-layer hydrate of sodium montmorillonite $(\mathrm{O}$ : red, $\mathrm{H}$ : white, $\mathrm{Si}$ : yellow, $\mathrm{Na}$ : blue, $\mathrm{Al}$ : purple, and $\mathrm{Mg}$ : green) with a charge of $-1.00 e$ per unit cell, representing the stable state at $T=298 \mathrm{~K}, p / p_{0}=0.3$. This simulation super cell consists of two interlayer regions made up by 8 unit cells. Molecular dynamics algorithms were applied to water and counterions. A temperature of $298 \mathrm{~K}$ was applied, while variations in the layer spacing were allowed under the influence of a constant stress perpendicular on these layers representing atmospheric pressure. Clay registration was incorporated according to the algorithms in ref 10 . Simulations were performed in the open ensemble with respect to clay water content. ${ }^{11,15}$ To this end, configurational-biased methods ${ }^{26}$ enabled efficient sampling of the number of adsorbed water molecules. The interaction potentials for the clay-water-counterion system are based on the TIP4P water model as outlined in ref 9 . These simulations essentially mimic the commonly applied water adsorption (desorption) experiment.

Our simulation strategy is to determine the average water content and layer spacing starting from different initial values 

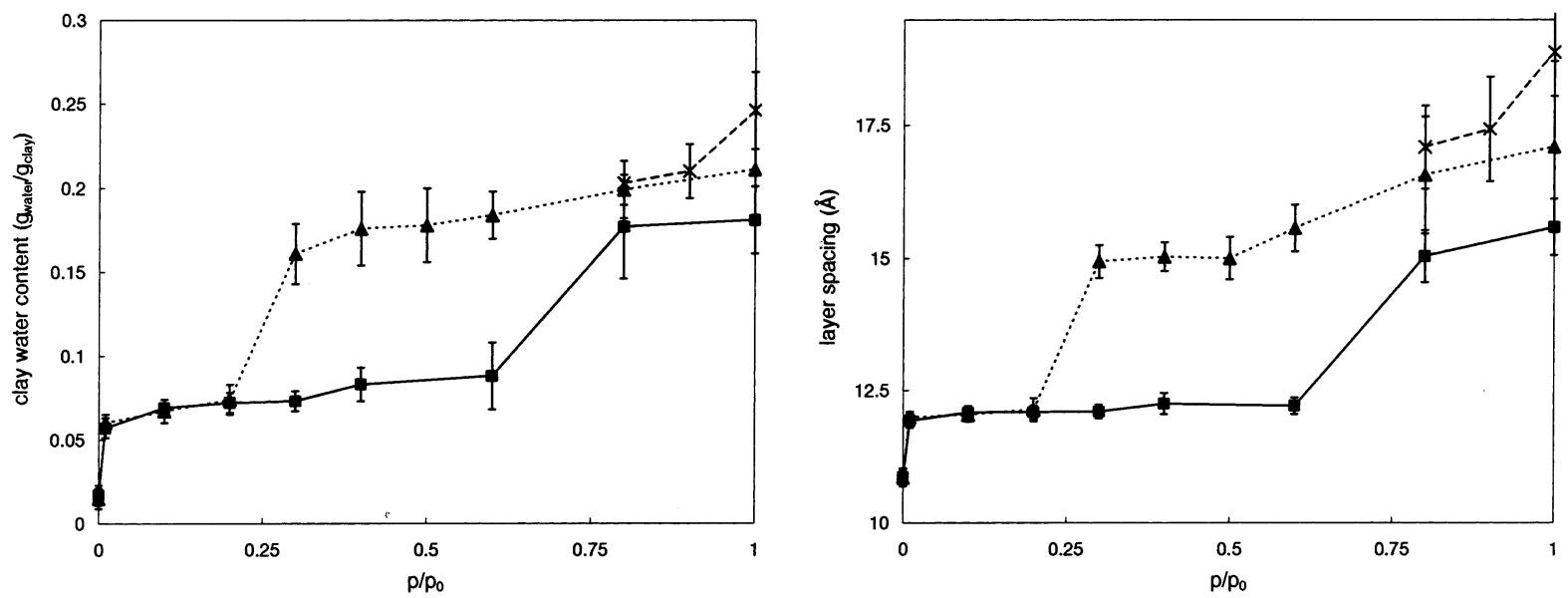

Figure 2. Computed swelling curves for sodium montmorillonite: clay water content (left) and layer spacing (right) as a function of the water vapor pressure. The various branches relate to different starting configurations (full line [ $\mathbf{\square}]: d_{0}=12.5 \AA$, $N_{0}=50$; dashed line - short [ $\left.\mathbf{\Delta}\right]: d_{0}$ $=15 \AA, N_{0}=100$; dashed line - long $\left.[\times]: d_{0}=17.5 \AA, N_{0}=150\right)$. The predicted stable states are close to experimentally observed ones for a dehydrated clay $(d \approx 10.0-10.4 \AA)$, a one-layer $(d \approx 12-12.5 \AA)$, two-layer $(d \approx 15 \AA)$, and three-layer hydrate $(d \approx 18-19 \AA)$. The integer numbers relate to the number of intercalated water layers.

for the number of water molecules per simulation super cell $\left(N_{0}\right)$ and the layer spacing $\left(d_{0}\right)$. Figure 2 displays the relation between the equilibrium layer spacing and clay water content as a function of the applied water vapor pressure for sodium montmorillonite. The most striking feature is the stepwise nature of the clay swelling process. One clearly observes the dehydrated state $(d=10.3 \AA)$, two well-defined hydrated states - a one- $(d$ $=12.3 \AA)$ and two- $(d=15.2 \AA)$ layer hydrate-and a lessdefined state $(d=16-18 \AA)$ at the highest water vapor pressure. This agrees well with the experimentally observed plateaus in layer spacing and clay water content. The values for the layer spacings of the three hydrates are close to experimental ones, whereas the computed interlayer water contents for the $n$-layer hydrates $\left(n=1: 0.060-0.090 \mathrm{~g} / \mathrm{g}_{\text {clay }} ; n=2: 0.160-0.190\right.$ $\mathrm{g} / \mathrm{g}_{\text {clay }}$, and $n=3: 0.200-0.250 \mathrm{~g} / \mathrm{g}_{\text {clay }}$ ) are marginally lower than experimental ones. ${ }^{16-21}$ This is partly due to known problems in determining experimental clay water contents (water adsorption on the external surface, presence of interstratified phases $^{21}$ ).

The first two stable hydrated states are well-defined in contrast to a third one identified at the highest water vapor pressures exhibiting strong fluctuations in layer spacing and water content. Such a third stable state has indeed been observed less frequently in experiments (e.g., in refs 16, 19, 20) than the first two. A further important point to note is that the computer simulations predict the occurrence of hysteresis in layer spacing and clay water content between the one- and two-layer hydrate. This phenomenon is often reported in smectite swelling measurements ${ }^{20,21}$ and derives from the mode of operation, that is, either allowing the clay to adsorb water or forcing water desorption. Our approach is similar, since the hysteresis phenomena result from starting at a small layer spacing with high water vapor pressure (adsorption) or starting at a large layer spacing with low water vapor pressure (desorption). Since the time and length scales in our simulations are much smaller than in a real experiment, we do not expect quantitative prediction of these hysteresis phenomena.

A careful analysis shows that the interlayer fluid structure coheres well with that described in earlier contributions. ${ }^{9,10,12}$ However, it disagrees with an earlier conclusion performed in the open ensemble ${ }^{11}$ which discounted the occurrence of a twolayer hydrate. The main differences with the present study are the use of the MCY interaction potential model and the absence of the Ewald summation technique to treat long-range electrostatic interaction. Most probably, the first factor is of overriding importance since the MCY water model is known to not correctly predict pressure.

\section{Mechanism of Clay Swelling}

The present algorithms provide a method to predict the stable states of swelling clays. However, ideally one would want to control the clay swelling in many engineered settings. Therefore, it is essential to understand the microscopic mechanisms underlying clay swelling. Such details are not easily amenable to experimental observation. Realizing that during our simulations transitions between the stable states occurred, we aimed to obtain exactly this kind of information. To this end, a simulation was performed, which started at the stable state corresponding to $p / p_{0}=0.3$, i.e., the one-layer hydrate. When the equilibrium state was reached, the water pressure was increased to $p / p_{0}=0.8$, corresponding to the two-layer hydrate. The water and sodium density profiles during swelling are condensed in Figure 3.

In the starting configuration, the one-layer hydrate is clearly visible with sodium ions forming inner-sphere surface complexes. ${ }^{4}$ In this regime, the $\mathrm{H}_{2} \mathrm{O} / \mathrm{Na}^{+}$ratio is between 3 and 4 . The water molecules are positioned in the center of the interlayer. The water oxygen coordinates to a sodium counterion, while the water hydrogen atoms hydrogen-bond to the clay surface oxygen atoms. With increasing water vapor pressure the clay swells but the inner-sphere complexes are preserved, their distance to the clay mineral surface remaining almost constant. Concomitantly, this forces the water molecules to form two layers. The increase in layer spacing results in less confinement of the water molecules allowing them to orient themselves to the sodium counterions. This is derived from the change in the angle between the water dipole and the clay mineral surface normal vector (Figure 3 ). Such a result can be interpreted as an increased orientation to the counterions and a decreased influence of hydrogen bonding to the clay mineral surface. It turns out that the water molecules close to one clay surface coordinate to sodium ions that are closest to the opposite one. The key phenomenon in the transition occurs at a layer spacing close to $13.50 \AA$ : part of the inner-sphere sodium ions move to the central plane of the interlayer. The driving force for this counterion flux is the energy associated with full 

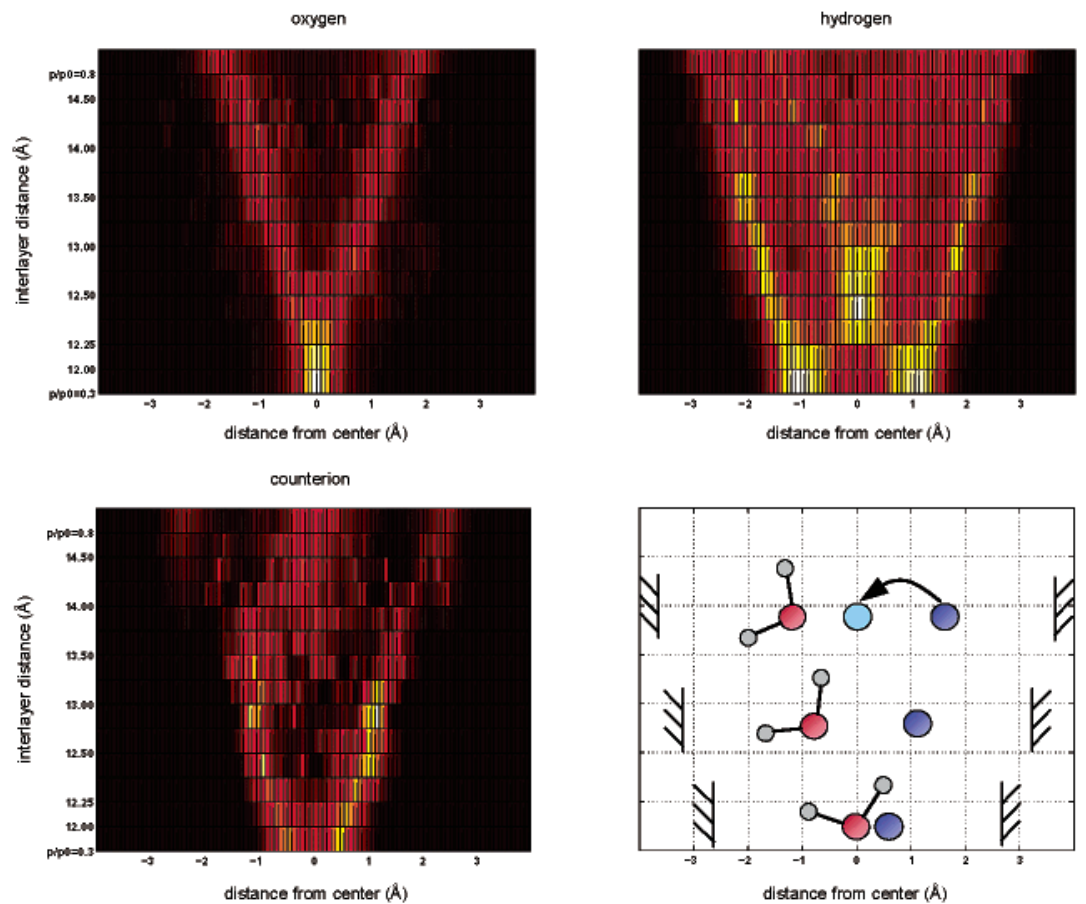

Figure 3. Evolution of the water and counterion distribution in the clay interlayer during the swelling transition. The graphs indicate the oxygen (upper left), hydrogen (upper right), and sodium (lower left) density as a function of the layer spacing (in bins of $0.25 \AA$ ). The integrated density at a given layer spacing is unity (color scheme: black-red-yellow-white from lowest to highest density). The splitting of the single water peak is observed at $d=12.5 \AA$, while the counterion flux to the central plane starts at approximately $13.5 \AA$. The lower right figure schematically shows the location and orientation of one counterion that remains close to the right clay mineral surface and coordinates to a water molecule closer to the opposite surface. The water molecule is subsequently reoriented, finally resulting in the transfer of the sodium ion from the inner-sphere position to the center of the interlayer. Note that only the position perpendicular to the clay surface is indicated; the clay mineral surface is also indicated.
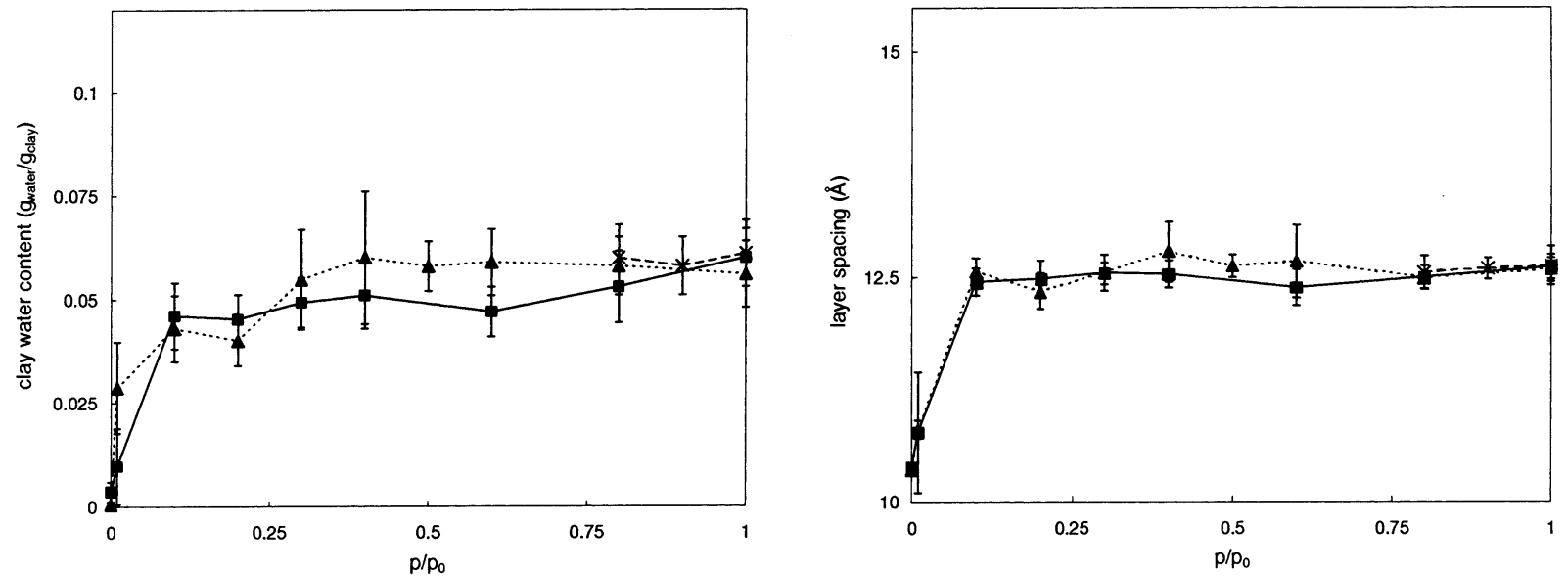

Figure 4. Computed swelling curve for sodium beidellite: clay water content (left) and layer spacing (right) as a function of the water vapor pressure. Beidellite only contains tetrahedral substitutions which strongly bind the counterions. This prevents counterions from becoming fully hydrated and the clay from swelling beyond the first stable state, even at the highest water vapor pressure. Note that for these simulations tetrahedral charge sites were implemented by randomly exchanging $\mathrm{Si}^{4+}$ sites by $\mathrm{Al}^{3+}$ sites, obeying Loewenstein's rule. No surface relaxation for tetrahedral charge sites or charge delocalization was allowed.

hydration of these counterions. The water molecules in this hydration shell interact with the clay mineral surface via hydrogen bonding, and these hydrated sodium ions are referred to as outer-sphere complexes. This hydration is facilitated by the increased interlayer volume and the increased number of intercalated water molecules. Notably, the sodium ions move to the interlayer center for a ratio $\mathrm{H}_{2} \mathrm{O} / \mathrm{Na}^{+} \approx 6$. This ratio agrees well with the sodium coordination number of concentrated $\mathrm{NaCl}$ aqueous solutions. ${ }^{22} \mathrm{~A}$ specific structural detail is that in the initial swelling stages the water molecules that are almost parallel to the clay sheets with their dipole vector are reoriented due to the increased counterion coordination. In this way, they position themselves in a favorable orientation for full hydration in the central interlayer plane. We suspect that this lowers the energy barrier for the transfer of sodium ions. A further expansion of the clay leads to an increasing number of sodium ions in the center of the interlayer and concomitant adsorption of water molecules that hydrate these ions. Particularly, one observes that in the regime 14.25-15.0 $\AA$ the water oxygen atoms overlap with the sodium ions that are located close to the inner-sphere positions. The resulting constraint is relieved by a further expansion to a layer spacing of about $15.3 \AA$, allowing a further increase of the clay water content and the formation of inner-sphere surface complexes. In this final state, a large fraction of counterions are fully hydrated outer-sphere complexes in the central interlayer plane. These observations 
indicate that the swelling proceeds from the state of partially hydrated inner-sphere sodium ions $\left(\mathrm{H}_{2} \mathrm{O} / \mathrm{Na}^{+} 3-4\right)$ to the state characterized by fully hydrated outer-sphere sodium ions with a smaller fraction of inner-sphere sodium ions $\left(\mathrm{H}_{2} \mathrm{O} / \mathrm{Na}^{+} \approx\right.$ 8-9). The essential transition takes place around $\mathrm{H}_{2} \mathrm{O} / \mathrm{Na}^{+}=$ 6. It may be envisioned that, in the final state, six water molecules make up the first coordination shell of the sodium ions located in the middle plane, while the remaining water molecules contribute to the formation of the smaller fraction inner-sphere surface complexes.

Until now, we considered a sodium montmorillonite model clay with only octahedral substitutions. Natural clay minerals such as the more common Wyoming-type montmorillonite clays have both octa- and tetrahedral substitutions, while beidellite presents the extreme case with only tetrahedral substitution. The cation-clay interaction strongly depends on the charge locus, tetrahedral charge sites-which are closer to the interlayerleading to more strongly bonded inner-sphere complexes. ${ }^{4}$ Figure 4 shows the computed water adsorption isotherms for sodium beidellite. The main result is the absence of a two-layer hydrate for the covered water vapor pressure range, even at the highest water vapor pressures. In this case, the sodium ions are so strongly bonded to the clay mineral surface (inner-sphere complexes) that they are not able to become fully hydrated in the central interlayer plane. Thus, the counterions cannot migrate to the interlayer center preventing the clay to swell. Indeed, it has been experimentally observed that beidellite does not easily swell beyond the one-layer hydrate in conventional swelling experiments. $^{23}$

Furthermore, it is well-known that clay swelling strongly depends on the type of the interlayer counterion. For instance, $\mathrm{K}^{+}$-containing clays show a lower tendency to swell than $\mathrm{Na}^{+}$containing ones, whereas the reverse is true for $\mathrm{Li}^{+}$-containing smectites. ${ }^{23,24}$ For potassium, the interaction with water is too weak, leading to the preferential formation of inner-sphere complexes independent of the location of the layer charge. This explains the efficiency of the use of $\mathrm{KCl}$ solutions as shale swelling inhibitors in drilling fluids. ${ }^{25}$ The stronger swelling tendency of Li-containing smectites may be related to the strongest hydration energy of the three discussed alkali ions. This would facilitate the transition of $\mathrm{Li}^{+}$at inner-sphere positions to the central plane.

\section{Concluding Remarks}

We have shown that a simple combination of molecular dynamics simulation of water and counterions between clay mineral surfaces and Monte Carlo sampling in the open ensemble allows one to quantitatively predict the clay water content of sodium montmorillonite. Moreover, it enabled us to obtain molecular insight into the transition of the one-layer to the two-layer hydrate for such a swelling clay. In essence, the results show that swelling proceeds by the migration of counterions initially strongly bound to the clay mineral surface to positions in the clay interlayer where these ions are fully hydrated. From these results, understanding of the dependence of clay swelling on charge locus, charge density, and the type of counterion can be derived.

\section{References and Notes} 177.

(1) Bucher, F.; Müller-Vonmoos, M. Appl. Clay Sci. 1989, 4, 157-

(2) Kajita, L. S. Clays Clay Miner. 1997, 45, 609-617.

(3) Zielke, R. C.; Pinnavaia, T. J.; Mortland, M. M. In Reactions and Movement of Organic Chemicals in Soils; Sawhney, B. L.; Brown, K., Eds.; Soil Science Society of America: Madison, 1989; pp 81-97.

(4) Sposito, G. The Chemistry of Soils; Oxford University Press: New York, 1989.

(5) Israelachvili, J. N. Surf. Sci. Rep. 1992, 14, 109-159.

(6) Bleam, W. F. Rev. Geophys. 1993, 31, 51-73.

(7) Delville, A. J. Phys. Chem. 1995, 99, 2033-2037.

(8) Chang, F.-R. C.; Skipper, N. T.; Sposito, G. Langmuir 1995, 11, 2734-2741.

(9) Boek, E. S.; Coveney, P. V.; Skipper, N. T. J. Am. Chem. Soc. 1995, 117, 12608-12617.

(10) Skipper, N. T.; Chang, F.-R. C.; Sposito, G. Clays Clay Miner. 1995, 43, 285-293.

(11) Karaborni, S.; Smit, B.; Heidug, W.; Urai, J.; Van Oort, E. Science 1996, 271, 1102-1104

(12) Chang, F.-R. C.; Skipper, N. T.; Refson, K.; Greathouse, J. A.; Sposito, G. In Mineral-Water Interfacial Reactions: Kinetics and Mechanism; Sparks, D. L., Grundl, T. J., Eds.; American Chemical Society: Washington, DC, 1999; pp 88-106.

(13) Young, D. A.; Smith, D. E. J. Phys. Chem. B 2000, 104, 91639170.

(14) Chávez-Páez, M.; Van Workum, K.; De Pablo, L.; De Pablo, J. J. J. Chem. Phys. 2001, 114, 1405-1413.

(15) Hensen, E. J. M.; Tambach, T. J.; Bliek, A.; Smit, B. J. Chem. Phys. 2001, 115, 3322-3329.

(16) Mooney, R. W.; Keenan, A. G.; Wood, L. A. J. Am. Chem. Soc. 1952, 74, 1371-1374.

(17) Norrish, K. Discuss. Faraday Soc. 1954, 18, 120-134.

(18) Cebula, D. J.; Thomas, R. K.; Middleton, S.; Ottewill, R. H.; White,

J. W. Clays Clay Miner. 1979, 27, 39-52.

(19) Kraehenbuehl, F.; Stoeckli, H. F.; Brunner, F.; Kahr, G.; MüllerVonmoos, M. Clay Miner. 1987, 22, 1-9.

(20) Fu, M. H.; Zhang, Z. Z.; Low, P. F. Clays Clay Miner. 1990, 38, 485-492.

(21) Cases, J. M. Langmuir 1992, 8, 2730-2739.

(22) Ohtaki, H.; Radnai, T. Chem. Rev. 1993, 93, 1157-1204.

(23) Newman, A. C. D. Chemistry of Clays and Clay Minerals; Mineralogical Society: London, 1987.

(24) Tamura, K.; Yamada, H.; Nakazawa, H. Clays Clay Miner. 2000, $48,400-404$.

(25) Denis, J. H.; Keall, M. J.; Hall, P. L.; Meeten, G. H. Clay Miner. 1991, 26, 255-268.

(26) Frenkel, D.; Smit, B. Understanding Molecular Simulation; Academic Press: San Diego, 1996. 